\title{
Copy number variations in endoglin locus: mapping of large deletions in Spanish families with hereditary hemorrhagic telangiectasia type 1
}

Ana Fontalba', Jose L Fernández-Luna', Roberto Zarrabeitia ${ }^{2}$, Lucia Recio-Poveda ${ }^{3}$, Virginia Albiñana ${ }^{3}$, Maria L Ojeda-Fernández ${ }^{3}$, Carmelo Bernabéu ${ }^{3}$, Luis A Alcaraz ${ }^{4}$ and Luisa M Botella ${ }^{3 *}$

\begin{abstract}
Background: The hereditary hemorrhagic telangiectasia syndrome (HHT), also known as the Rendu-Osler-Weber syndrome is a multiorganic vascular disorder inherited as an autosomal dominant trait. Diagnostic clinical criteria include: epistaxis, telangiectases in mucocutaneous and gastrointestinal sites, arteriovenous malformations (AVMs) most commonly found in pulmonary, hepatic and cerebral circulations, and familial inheritance. HHT is transmitted in $90 \%$ of the cases as an autosomal dominant condition due to mutations in either endoglin (ENG), or activin receptor-like kinase 1 (ACVRL1/ALK1) genes (HHT type 1 and 2, respectively).

Methods: We have carried out a genetic analysis of four independent Spanish families with HHT clinical criteria, which has permitted the identification of new large deletions in ENG. These mutations were first detected using the MLPA technique and subsequently, the deletion breakpoints were mapped using a customized copy number variation (CNV) microarray. The array was designed to cover the ENG gene and surrounding areas.

Results: All tested families carried large deletions ranging from 3-kb to 100-kb, involving the ENG gene promoter, several ENG exons, and the two downstream genes FGSH and CDK9. Interestingly, common breakpoints coincident with Alu repetitive sequences were found among these families.

Conclusions: The systematic hybridization of DNA from HHT families, with deletions or duplications, to custom designed microarrays, could allow the mapping of breakpoints, coincident with repetitive Alu sequences that might act as "hot spots" in the development of chromosomal anomalies.
\end{abstract}

Keywords: Hereditary hemorrhagic telangiectasia (HHT), Endoglin deletions, Multiplex ligation PCR assisted assay (MLPA), Copy number variation (CNV) arrays, Alu repetitive sequences

\section{Background}

The hereditary hemorrhagic telangiectasia syndrome (HHT), also known as Rendu-Osler-Weber syndrome [1-3] is a vascular disorder inherited as an autosomal dominant trait. Careful epidemiological studies have revealed that HHT affects approximately 1 in 5,000 individuals [4,5] and is therefore considered to be an inherited rare vascular disease.

\footnotetext{
* Correspondence: cibluisa@cib.csic.es

${ }^{3}$ Centro de Investigaciones Biológicas, Consejo Superior de Investigaciones Científicas (CSIC), and Centro de Investigación Biomédica en Red de Enfermedades Raras (CIBERER), Madrid, Spain

Full list of author information is available at the end of the article
}

The clinical symptoms characteristic of HHT are the so-called Curaçao criteria [2], which help in its diagnosis when at least 3 of the 4 criteria are present in a patient. These include: epistaxis (nose bleeds), telangiectasia at mucocutaneous and gastrointestinal sites, arteriovenous malformations (AVMs) most commonly found in pulmonary, hepatic and cerebral circulations, and dominant familial inheritance $[3,6]$.

HHT is transmitted as an autosomal dominant condition due to a single mutation in Endoglin (ENG; HHT1) [7], Activin Receptor-Like Kinase 1 (ACVRL1/ALK1; HHT2) [8], or MADH4/SMAD4 (JHPT, a combined syndrome of juvenile polyposis and HHT) [9]. The involvement of all these genes in the transforming growth factor $(\mathrm{TGF}-\beta)$ 
signaling pathway is inherent in HHT pathogenesis [10]. There are at least two further unidentified genes that can cause HHT: HHT3 between 141.9 and 146.4 Mb on chromosome $5 \mathrm{q}[3,11]$ and HHT4 on chromosome $7 \mathrm{p}$ between D7S2252 and D7S510.130 [12].

The genes mutated in HHT encode proteins that mediate signaling by the TGF- $\beta$ superfamily. Members of this superfamily such as TGF- $\beta$ s, bone morphogenetic proteins (BMPs), activins, nodals, growth/differentiation factors (GDFs) and inhibins regulate diverse cellular functions by binding to a heteromeric complex of type I and type II transmembrane serine/threonine kinase receptors [13]. In the TGF- $\beta$ signaling cascade, the type II receptor with very high ligand affinity, co-operatively recruits and transphosphorylates the type I receptor by direct contact with the ligand-modified N-terminus of T $\beta R I$ [14]. In Smad-dependent TGF- $\beta$ pathways, the type I receptor subsequently phosphorylates and activates receptor-associated (R)-Smads, according to the receptor complex involved. R-Smads bind to Smad4 and translocate to the nucleus where they influence transcriptional activity with co-activators and co-repressors.

In endothelial cells, upon ligand binding, T $\beta$ RII can associate with two different TGF- $\beta$ type I receptors ALK-5 or ALK-1 [15]. Endoglin is an auxiliary receptor that modulates both associations in an opposite manner. Thus, while endoglin promotes signaling through ALK1 , it inhibits the ALK-5 pathway [16,17]. In turn, ALK-1 and ALK-5 activate distinct Smad pathways, resulting in opposing endothelial cell responses in terms of proliferation, migration, and pro- or anti-angiogenic gene expression $[15,18,19]$.

So far, more than 600 different mutations have been found in ENG and ACVRL1 in HHT families (HHT mutation database; http://www.arup.utah.edu/database/hht/). Mutations range from single base-pair changes to major deletions of multiple exons. Recently, pathogenic mutations affecting the 5' UTR region of ENG leading to new translation initiation sites (TIS) dominant over the normal endoglin TIS have also been described [20,21].

In the present study we describe for the first time, a series of 4 families, harboring 4 different independent mutations in the ENG gene, not described so far in literature. All of them were large deletions starting in the 5 ' upstream region of ENG, and spanning just the promoter region, one or several exons, or even a big $100-\mathrm{Kb}$ deletion encompassing the whole ENG gene and the two downstream genes FGSH and CDK9. These mutations were first detected by MLPA technique, and subsequently the breakpoints were characterized using a customized copy number variation (CNV) microarray, designed to cover ENG and flanking sequences. Interestingly, common breakpoints located within "Alu" sequences were found among these families. To the best of our knowledge, this is the first time a fine mapping of a series of deletion mutations is described in HHT taking advantage of a customized $\mathrm{CNV} / \mathrm{CGH}$ microarray.

\section{Methods \\ Patient samples}

Blood samples of 4 independent families from Spain with clinically confirmed HHT (the presence of three or four Curaçao criteria) were subjected to molecular diagnostics. The clinical data of these patients were obtained from screening performed in the Spanish HHT reference Center of Sierrallana, or alternatively from the referring physicians. Informed consent was obtained from all the patients.

\section{Ethics statement request}

Human blood samples and clinical data reported in this manuscript were obtained with the prior approval of the appropriate ethics committees of the CSIC for the research conducted in the Centro de Investigaciones Biológicas (CIB, Madrid), the CEIC (Clinical Research Ethics Committee) of the Cantabrian Health Service for patients attending the Hospital of Sierrallana (Torrelavega, Santander, Cantabria), and the Medical Genetics department of the Hospital Valdecilla (Santander, Cantabria). Research was carried out in compliance with the Helsinki Declaration (http:// www.wma.net/en/30publications/10policies/b3/index.html), keeping the results strictly confidential, with number codes for the identification of patients.

\section{DNA extraction and mutation analysis}

DNA was extracted from peripheral blood using standard procedures. Each coding exon and its flanking intronic sequences were amplified by PCR and sequenced for each of the two genes (ENG and ACVRL1). Primer sequences and PCR conditions have already been reported $[10,22]$ and are available upon request. PCR products were run on $1.5 \%$ agarose $/ 1 \times \mathrm{TAE}$ gels and purified using a PCR purification kit (Millipore, Germany). The PCR products were then sequenced in forward and reverse orientation on an Applied Biosystems sequencer using the dye terminator cycle sequencing kit according to the manufacturer's instructions. If no mutation was found either in ENG or ALK1, MLPA was performed. DNA concentration was measured for each sample using a Nanodrop spectrophotometer. At the same time the DNA integrity was checked by agarose gel electrophoresis $(0.8 \%$ in TAE).

Multiplex ligation-dependent probe amplification (MLPA) MLPA [23] was performed with $200 \mathrm{ng}$ of genomic DNA according to the manufacturer's instructions using the P093 Salsa MLPA HHT/PPH1 probe set (MRC-Holland, Amsterdam, The Netherlands). Probe amplification products were run on an ABI PRISM 310 Genetic Analyzer using 
GeneScan-500 TAMRA Size Standard (PE Applied Biosystems). Data were analyzed using Coffalyser MLPA data analysis software, and a relative copy number was obtained after normalization of peaks against controls. Values between 0.75 and 1.3 were considered to be within the normal range. (LLC, State College, PA). Because of variations in assay performance, we used dosage ratio values of $\leq 0.7$ as our boundaries.

\section{Characterization of the breakpoints}

Breakpoints were studied using a custom designed CGH microarray (Agilent) to detect copy number variations. A tiling microarray was designed with eArray (Agilent). The array contains a total of 15,744 probes, including: i) 7,568 probes of $E N G$; ii) 3,927 probes from the rest of chromosome 9; iii) 1,262 probes included for normalization; and iv) 2,987 probes as Agilent controls. A total of 7,568 probes were selected in the region of interest (chr9: 130548305-130661871, hg19) covering the ENG gene and the surrounding upstream and downstream sequences to complete a total area of $113-\mathrm{Kb}$, with the ENG gene in its center (Figure 1). Probes were designed with an average probe spacing of 15-bp, and allowed to vary in size (from 45 - to 60 -mer) in order to maintain their Tm about $80^{\circ} \mathrm{C}$. The rest of the chromosome 9 was covered with 3,927 additional probes. For normalization purposes during data analysis, 1,262 probes, distributed among all chromosomes, were also included. 2,987 probes were Agilent controls. A probe performance score was assigned to each probe by the software.

Labeling was carried out according to manufacturer protocol 1 (Agilent Oligonucleotide Array-Based CGH for Genomic DNA Analysis, v6.3). Briefly, eight genomic DNA samples, including four controls (non HTT patients), were Cy5 labeled and co-hybridized with a sex matched, Cy3 labeled human genomic reference DNA (Promega). After hybridization, arrays were washed and the signal for each probe was captured (scanned) with an Agilent scanner.
Images were analyzed using Agilent Feature Extraction v10.7 software. The quality of the hybridization was estimated by the DLRS parameter, indicating the dispersion among the hybridization signaling of the probes. In all cases, good DLRS values, between 0.1 and 0.2 were obtained.

Finally, raw data were analyzed with Agilent Genomic Workbench 6.5. Pre-processing steps were done by applying GC correction and centralization. Probes with scores below 0.5 were filtered out. Aberration calling was done with the ADM-2 algorithm with a threshold of 0.6. An aberration was considered when there were at least 5 consecutives probes with a minimum absolute average log ratio of 0.25 . The breakpoint regions were inspected with a UCSC Genome Browser, using the Repeat Masker database to identify repeated elements.

\section{Results}

\section{Correlation between the presence of deletions in ENG} and the clinical phenotype

Table 1 shows the clinical symptoms and genetics of HHT patients belonging to 4 independent families. The clinical symptoms are always accompanied by the presence of the mutations commented on below. Those patients with known genetics, but referenced in Table 1 with "no data" for the different clinical HHT symptoms, had not undergone a complete clinical screening, whereas patients with complete data were either, screened in the Spanish HHT Reference Center of Sierrallana, or elsewhere. In these families, there was a perfect phenotypegenotype correlation between clinical criteria and the finding of mutations in ENG. Exceptions to this rule were children and teen-agers, most of them without relevant epistaxis, with no telangiectases, and not subjected to the complete HHT protocol of clinical screening. Close relatives with no clinical symptoms were also genetically tested.

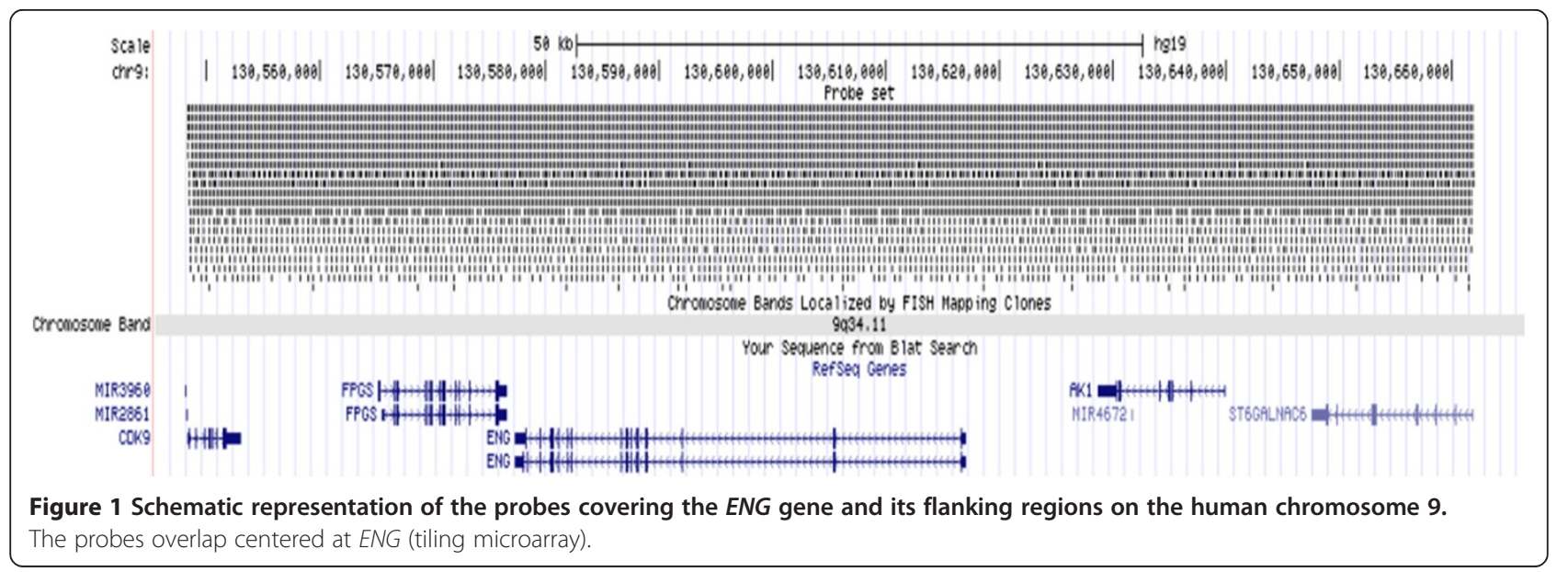


Table 1 Genotype-phenotype correlation in 4 families with HHT

\begin{tabular}{|c|c|c|c|c|c|c|c|}
\hline Family & Epistaxis & Telangiect & PAVMs & CAVMs & HAVMs & GB & Genetics \\
\hline \multicolumn{8}{|l|}{ NMEx } \\
\hline JCG & Yes & Yes & Yes & No & No & No & $\Delta$ Promoter ENG \\
\hline PCG & Yes & Yes & No & No & No & No & $\Delta$ Promoter ENG \\
\hline MJCS & Yes & Yes & Yes & No & No & No & $\Delta$ Promoter ENG \\
\hline VAC & Yes & Yes & No & No & No & No & $\Delta$ Promoter ENG \\
\hline \multicolumn{8}{|l|}{198} \\
\hline 198/01/11 & Yes & Yes & Yes & No & No & No & $\Delta$ Promoter + exon 1 ENG \\
\hline 198/02/11 & Yes & Yes & No data & No data & No data & No data & $\Delta$ Promoter + exon 1 ENG \\
\hline 198/03/11 3 y & No & No & No data & No data & No data & No data & $\Delta$ Promoter + exon 1 ENG \\
\hline \multicolumn{8}{|l|}{71} \\
\hline $71 / 02 / 08$ & Yes & Yes & Yes & No & Yes & Yes & $\Delta$ Promoter + exons 1-2-3 ENG \\
\hline $71 / 03 / 11$ & No data & No data & No data & No data & No data & Yes & $\Delta$ Promoter + exons 1-2-3 ENG \\
\hline $71 / 04 / 11$ & No data & No data & No data & No data & No data & No data & $\Delta$ Promoter + exons 1-2-3 ENG \\
\hline $71 / 08 / 1113$ y & No & No data & Yes & No data & No data & No data & $\Delta$ Promoter + exons 1-2-3 ENG \\
\hline $71 / 09 / 1114$ y & Yes & No data & Yes & No data & No data & No data & $\Delta$ Promoter + exons 1-2-3 ENG \\
\hline $71 / 12 / 116$ y & No & No data & No data & No data & No data & No data & $\Delta$ Promoter + exons 1-2-3 ENG \\
\hline \multicolumn{8}{|l|}{ GUM } \\
\hline MCDV & Yes & Yes & Yes & Yes* & No & No & \#Promoter and complete allele ENG ( -9 kb + all exons) \\
\hline$J M V D$ & Yes & Yes & No data & No data & No data & No data & \#Promoter and complete allele ENG ( -9 kb + all exons) \\
\hline $\mathrm{JDHV}$ & Yes & Yes & No data & No data & No data & No data & \#Promoter and complete allele ENG ( -9 kb + all exons) \\
\hline
\end{tabular}

E, stands for epistaxis, and T for telangiectases. PAVM, CAVM, HAVM stand for pulmonary, cerebral and hepatic arteriovenous malformations, respectively. GB means gastric bleeding. *patient MCDV had a brain abscess in addition to CAVMs. $\Delta$ stands for deletion. \# stands for complete deletion of gene.

\section{Family NMEx}

Family NMEx, comes from Navalmoral de la Mata, a town in Extremadura, western Spain. These patients were referred to our HHT unit from the local hospital of this town, because they presented epistaxis, telangiectasia, and some of them also suffered from internal arteriovenous malformations (Table 1). After amplification and sequencing of all exons and intron-exon boundaries of ENG and ACVRL1 genes, no mutation was found. Therefore, according to the protocol established in our HHT unit, an MLPA was performed. By comparing patient JCG with a control sample (Figure 2A), it can be seen that only the peak corresponding to the ENG MLPA probe annealing in the proximal promoter region appears affected. The same result was obtained for the four members of this family, whose clinical data are listed in Table 1, while relatives without HHT criteria, did not show any alteration in the MLPA (data not shown). Notably, no mutation involving a deletion of the ENG promoter has been reported in HHT so far. The possible existence of a polymorphism in the hybridization area of the corresponding MLPA probe, which could prevent primer annealing, was discarded by sequencing the proximal promoter region, from $-450 \mathrm{bp}$ to the transcription start (data not shown).

\section{Family 198}

In this family from northern Spain, a total of 3 members with clinical diagnosis of HHT were subjected to the normal protocol of PCR amplification and sequencing, but no mutations in either ENG or ACVRL1 were observed. As in the case of family NMEx, MLPA analysis was carried out, and a deletion involving the promoter and exon 1 of ENG was found. In Figure 2A, bottom panel, a representative chromatogram of an affected family member (198/01/11) reveals that peaks corresponding to promoter and exon 1 are both decreased in comparison with a control DNA.

\section{Family 71}

A total of 6 members, 3 adults and 3 children, were genetically screened. All three adults fulfilled the clinical Curaçao criteria. As in previous families, while the PCR and sequencing did not reveal any point mutation or short duplication/deletion, the MLPA showed a large deletion encompassing the promoter and exons 1 to 3 of ENG (Figure 2B). Moreover, the 3 children had no visible clinical signs of HHT, but their positive genetic test result prompted a subsequent, in depth clinical examination and led to the finding of pulmonary AVMs in two of them. 


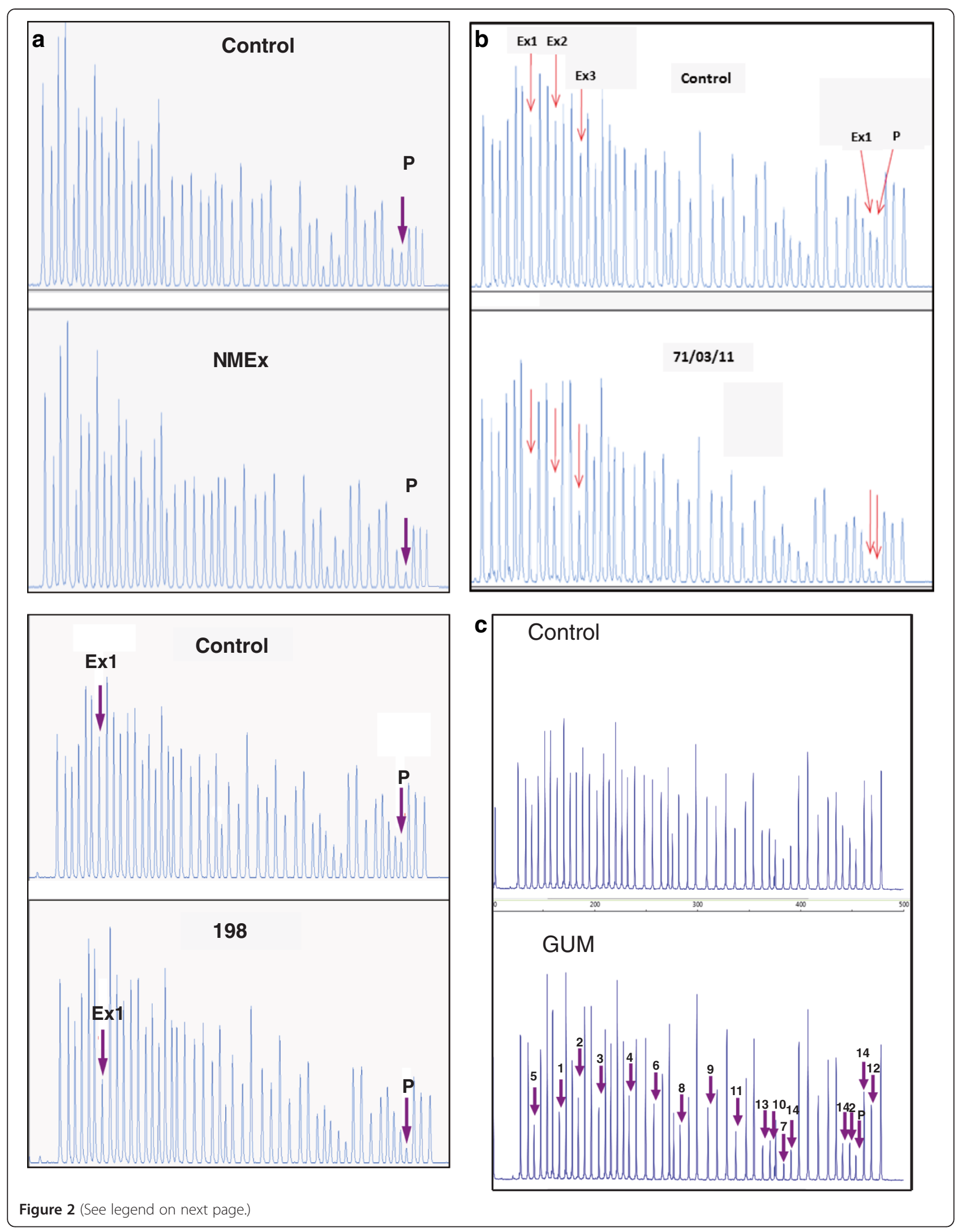


(See figure on previous page.)

Figure 2 MLPA results of the 4 different families analyzed in the present work. A) Control and chromatogram representing the family with promoter deletion (NMEX) and with promoter and exon 1 deletion (198). B) Control chromatogram and family 71 exhibiting deletion of promoter and exons from 1-3. C) Family GUM with whole deletion of ENG gene including the promoter. In the chromatograms the height of the peaks is proportional to the amplification of the corresponding exon probe. The affected exons are indicated by arrows in each case.

\section{Family GUM}

Three members of a family from Madrid were diagnosed with clinical criteria of HHT. The conventional genetic analysis did not find any mutation, but MLPA analysis (Figure 2C), revealed the complete absence of one ENG allele starting at $9 \mathrm{~kb}$ upstream from the transcription start site, suggesting that these patients were hemizygous, at least for $E N G$.

\section{Characterization of the breakpoints for the ENG deletion mutants}

In all these families there were common properties that made them especially interesting for further analyses: all of them contained deletions in ENG, not previously published in HHT literature, and all these deletions started on one side of the promoter region of ENG. Thus, it was of interest to analyze: i) the mapping of the different deletions; ii) whether there were common breakpoints; and iii) whether there was a common characteristic shared by these regions prone to breaking. Accordingly, breakpoints were studied with a custom designed tiling CGH microarray (Agilent) to detect copy number variations.

A schematic representation of the final deletion map, for each family, and the genes affected is shown in Figure 3.

A more detailed view of the individual hybridization of each DNA sample to the probes of the array can be seen in Additional file 1: Figure S1. The upper part of the panels in Additional file 1: Figure S1 represents a general view of the hybridization signals within the 9q34.11 region. At the bottom, a zoom of the region containing the copy number alteration is presented in more detail. A shadow box over a crowd of points represents the area where the algorithm has detected a copy number alteration. A minimum of 5 probes, hybridizing in the same manner and placed in the same area, are required to define a significant copy number alteration over a chromosome position.

Table 2 shows a summary of the results obtained from the microarray, showing the exact breakpoints for each deletion found in the four different families. Deletions affecting coding regions of ENG include: i) the smallest deletion of $3,139-\mathrm{Kb}$, affecting the proximal promoter and exon 1 of ENG (family 198); ii) an intermediate size deletion encompassing $28,744-\mathrm{Kb}$, starting at the same point of the proximal promoter as the previous one, and spanning exons 1 to 3 of ENG (family 71); and iii) the largest deletion, spanning from $9 \mathrm{~kb}$ upstream from the transcription start site of ENG, the whole ENG gene, and two adjacent ENG-downstream genes FPGS, CDK9 and, at least, partially $S H 2 D 3 C$ (family GUM). Furthermore, a deletion affecting only the upstream region of $E N G$, which does not include any coding region, comprises $9,313-\mathrm{Kb}$, from the same position as in the case of the largest deletion of the GUM family in the distal promoter region to position -28 bp before the transcription start for ENG (family NMEx).

Interestingly enough, these 4 different deletions have some breakpoints in common: two of them start 9.3-Kb upstream of the ENG transcription start site, and the other two start approximately 900-bp upstream from the

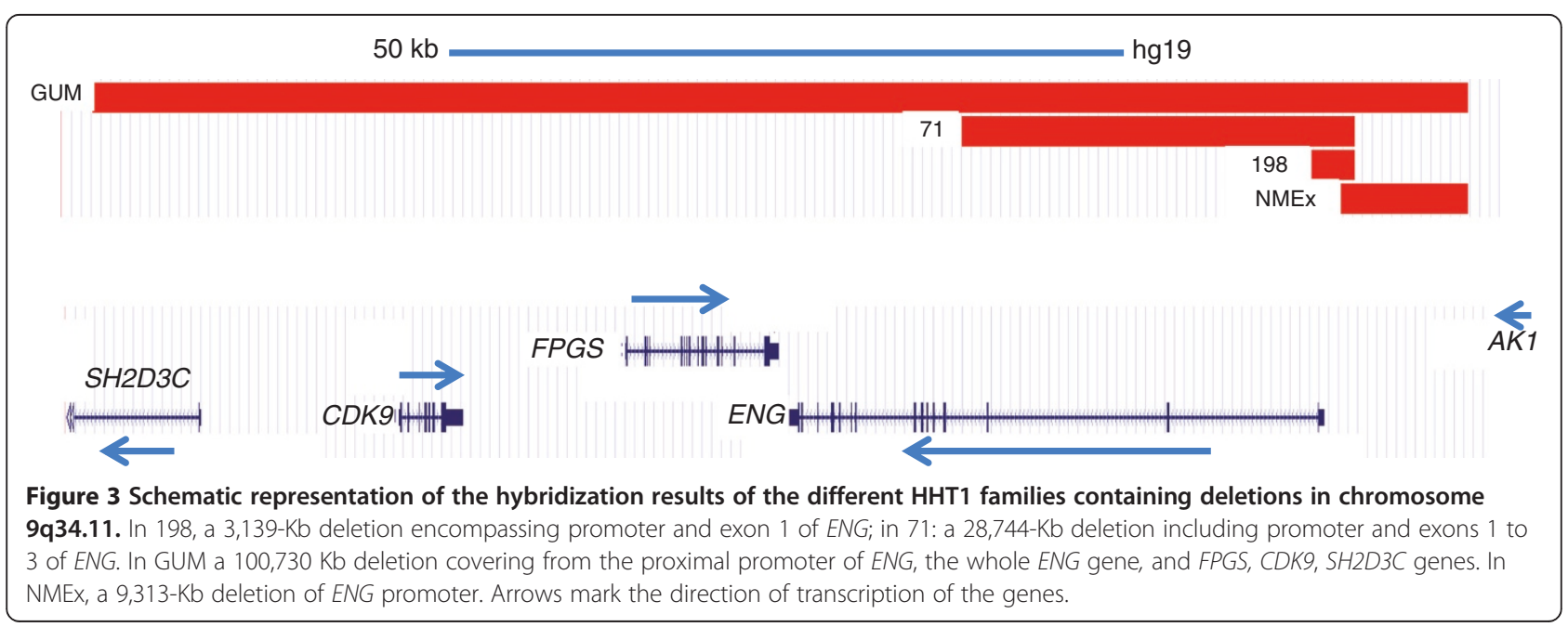


Table 2 Deletion break-points for HHT families

\begin{tabular}{lllllll}
\hline Sample & Cytoband & Ins/Del & Molecular position & Size & Regions deleted & Affected genes \\
\hline JGC & $9 q 34.11$ & Del & $130,617,254-130,626,567$ & $9,313 \mathrm{~Kb}$ & Promoter 5' upstream ENG & Promoter ENG \\
198/01/11 & $9 q 34.11$ & Del & $130,615,100-130,618,239$ & $3,139 \mathrm{~Kb}$ & Promoter and Exon 1 ENG & ENG \\
71/03/11 & $9 q 34.11$ & Del & $130,589,495-130,618,239$ & $28,744 \mathrm{~Kb}$ & Promoter and Exon 1-3 ENG & ENG \\
MCVD & $9 q 34.11$ & Del & $130,525,837-130,626,567$ & $100,730 \mathrm{~Kb}$ & $9.3 \mathrm{~Kb}$ promoter 5' upstream, & SH2D3C, FPGS, CDK9C ENG \\
& & & & & whole ENG and surroundings & \\
\hline
\end{tabular}

transcription start site. These findings support the existence of "hot-spots" for chromosomal rearrangements in these regions.

To characterize the nature of these regions prone to "breaking", we studied the sequences surrounding the common breakpoints for the deletions, on the assumption that regions containing repeated elements are more likely to break. In order to get more insight into the sequence structure of the breakpoints we analyzed these regions in more detail with the Repeat Masker tool for repeat sequences of the UCSC Genome Browser. Figure 4A shows different repeat elements around the breakpoint common to families 198 and 71. Among them, the Alu element AluSz, that contains the common breakpoint, is located 900-bp upstream of the transcription start, and was first described for the ENG promoter by Rius et al. [24].

The other breakpoint, found in the remaining two families (GUM and NMEx), is located further upstream from the ENG promoter. In this case, there is an accumulation of repeat elements surrounding the breakpoint, including mostly Alu sequences and also a LINE1 element (L1ME2z), as shown in Figure 4B. These results suggest that breakpoints upstream of ENG are positioned where mobile or repeated elements are located.

\section{Discussion}

Most of the mutations involved in the pathogenesis of HHT are single base-pair substitutions or small duplications/

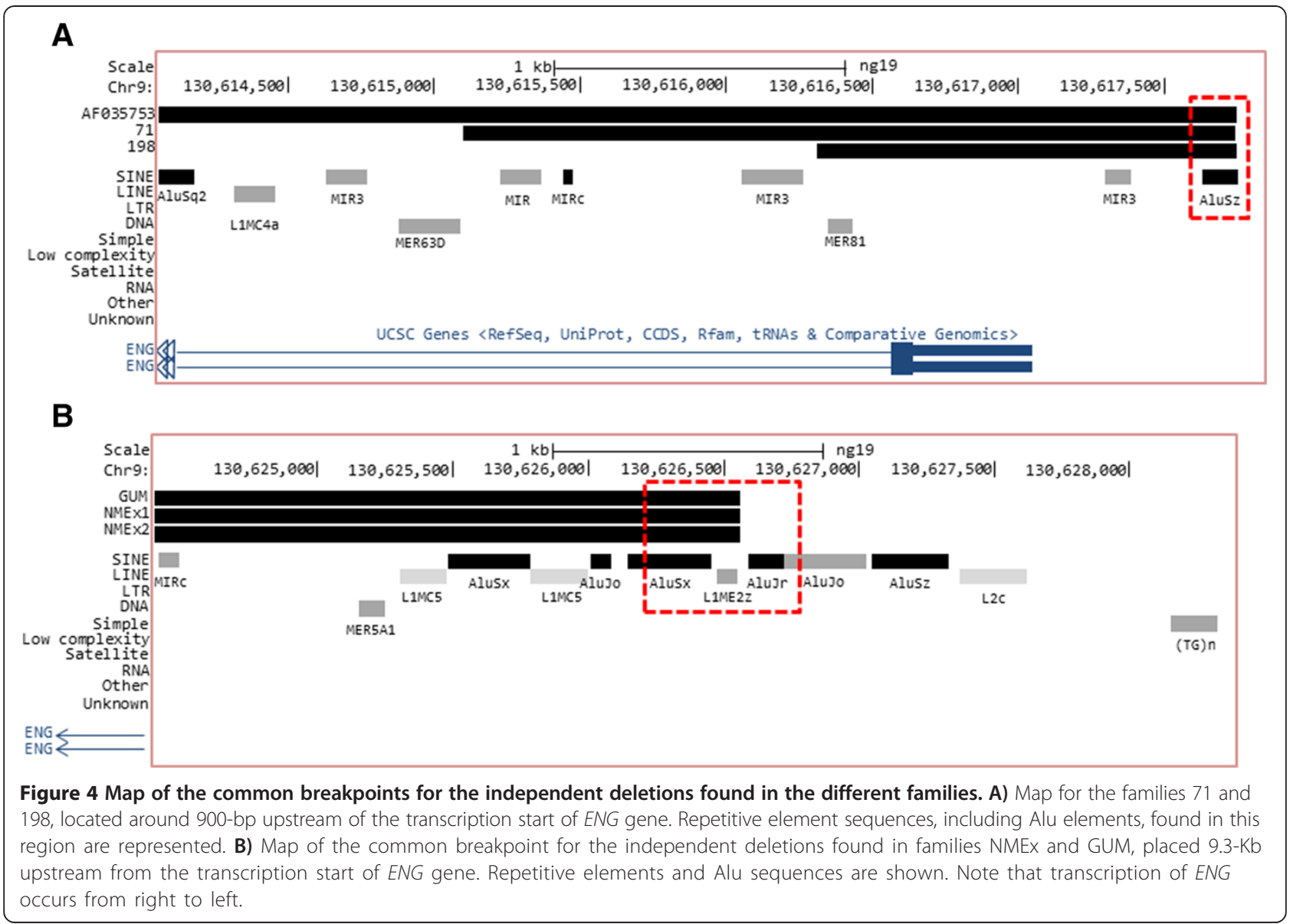


deletions. In fact, a review of molecular diagnosis of HHT identified for ENG, 17\% nonsense, 30\% missense, $25 \%$ splice, and $28 \%$ frameshift mutations, whereas for ACVRL1 17\% were nonsense 60\% missense, $7 \%$ splice, and $15 \%$ frameshift mutations [25].

When no mutations are found in either ENG or ACVRL1 by PCR and sequencing of coding exons and intron boundaries, then the multiplex quantitative PCR (qPCR)-based assay method MLPA is the standard routine technique applied to samples. MLPA allows the identification of dosage for proximal promoter and exons in the HHT-related genes, including deletions or duplications of whole exons. However, this method does not yield a systematic mapping of the breakpoints corresponding to these chromosomal aberrations. So far, mutations affecting the copy number variation of ENG or ACVRL1 causing HHT, as evidenced by MLPA, represent around $7 \%$ of all the pathogenic mutations detected in these genes [25,26].

More recently, CNV custom CGH arrays have emerged as an alternative technique to MLPA to detect large deletions or insertions, with the advantage of allowing the mapping and detailed analysis of breakpoints. Thus, this technique can reveal the presence of "hot spots" or points prone to breaking, which may lead to common mutation events in independent families, and coincident with repetitive sequences. In the present work we have identified and mapped four large deletions affecting ENG that have not been previously reported in publications and which were first detected by MLPA. To the best of our knowledge, this is the first time that the breakpoints of large deletions in ENG have been mapped in independent HHT families.

In all cases the deletions started in the region of the ENG promoter and were the first reported deletions affecting the promoter and implicated in HHT. Interestingly, the affected members of the family NMEx were heterozygous for a $9-\mathrm{Kb}$ deletion of the promoter expanding up to $28 \mathrm{bp}$ upstream of the transcription initiation start site. In this case, the loss of the promoter region leading to a hemizygous allele is the likely cause of HHT. Indeed, the proximal promoter region of ENG plays a critical role in the basal transcription, mainly through binding of the transcription factor Sp1 to consensus GC-rich motifs [24,27]. Additionally, the promoter activity of ENG can be regulated by several physiological stimuli. In this regard, Sp1 can directly bind, in protein-DNA complexes, to the transcription factors KLF6, Smad3/Smad4 or HIF$1 \alpha$, activated by vascular injury [28], treatment with TGF- $\beta 1$ [27] or hypoxia [29], respectively. In turn, the multicomplex formed by all these transcription factors leads to synergistic transcriptional cooperation with the promoter activity of ENG. Therefore, basal and stimuli-dependent transcription of ENG would be abrogated in those HHT patients harboring a promoter deletion, contributing to the haploinsufficiency of ENG.

HHT patients from family GUM are hemizygous not only for ENG, but also for the genes FPGS, CDK9 immediately downstream, and at least part of $S H 2 D 3 C$. However, the additional heterozygous deletion of these genes does not seem to affect the severity or type of clinical symptoms. This would explain the lack of a reported pathology associated with heterozygous mutations of these genes in relevant scientific publications.

The detailed mapping of the deletions has revealed interesting hot spots in ENG introns and their upstream promoter region where common breakpoints have been found. Thus, we have identified two different breakpoints placed in non-coding regions corresponding to allocation sites for Alu mobile elements, and both derived from independent recombination events in different families. One of the breakpoints is within a cluster of Alu sequences, 9-Kb upstream of the ENG transcription start site (Figure 4B), and the other is on an Alu element, placed $900 \mathrm{bp}$ upstream of the ENG transcription start site (Figure 4A). In agreement with this finding, Wooderchak et al. [30] described the breakpoints for two deletions affecting ENG in a single HHT family, one of them encompassing exon 3 and the other involving exons 4 to 7 [30]. Interestingly, both deletions share a common breakpoint location in intron 3. Furthermore, a large 117-bp repetitive DNA sequence was identified near the breakpoints in introns 2, 3, and 7 of ENG. These repetitive sequences had a sequence identity of approximately $85 \%$, had similar orientation, and were each found to contain Alu elements. These results suggest that this type of mobile element is a common target of recombination in HHT genes. Supporting this view, Alu sequences have been involved in the generation of genomic deletions in different human genetic disorders [31,32]. Further studies have yet to be carried out to better understand the mechanisms of recombination in HHT. In this regard, it would be interesting to screen the published deletions and duplications found in ENG and $A C V R L 1$, taking advantage of the $\mathrm{CNV}$ array technique to obtain a map of deletions or duplications from as little as 15-bp to many kilobases.

\section{Conclusions}

The systematic hybridization of DNA from HHT families, with deletions or duplications, to custom designed microarrays, could allow the mapping of breakpoints, coincident with repetitive Alu sequences that might act as "hot spots" in the development of chromosomal anomalies. New data, generated this way, would then enrich the HHT mutation databank by including the specific sequences of the chromosomal rearrangements, in addition to the current mutations. 


\section{Additional file}

Additional file 1: Figure S1. Hybridization results of the different DNAs to the CGH/CNV array. A) Hybridization results from sample 198/01/11: 3,139-Kb deletion chromosome 9q34.11: promoter and exon 1 of ENG. B) Hybridization results from sample 71/03/11, a 28,744-Kb deletion chromosome 9q34.11, including promoter and exons 1 to 3 of ENG. C) Hybridization results from sample GUM: 100,730-Kb deletion chromosome 9q34.11: promoter ENG, FPGS, CDK9 and SH2D3C genes. D) Hybridization results from sample NMEx: 9,313-Kb deletion chromosome 9q34.11: promoter of ENG. In all cases, the general view of chromosome 9 with the probe hybridization is presented above, and below the region containing the deletion is magnified to show details.

\section{Competing interests}

The authors declare that they have no competing interest.

\section{Authors' contributions}

AF: most sequencing and MLPA. JLF-L: direction of the sequencing and MLPA. RZ: clinician of the hospital center for HHT. LR-P: DNA extraction, PCRs, probe preparation. VA: DNA extraction, and PCR. MLO-F: DNA extraction and PCR. CB: part of the funding support, edition of Ms. LAA: hybridization of CNV arrays. "LMB: direction and coordination of experiments, design and experimental approach strategies. Project fund provision. Writing the manuscript. All authors read and approved the final manuscript.

\section{Acknowledgments}

This work was supported by grants from Ministerio de Economia y Competitividad (SAF2008-01218 and SAF2011-23475 to LMB; and SAF201019222 to CB), CIBERER (Intramural 11-707/112.02) and Fundación Ramón Areces (FRA; Rare and Emergent Diseases to LMB) of Spain. Virginia Albiñana was supported by FRA. Maria L. Ojeda-Fernandez is recipient of a CIBERER contract. CIBERER is an initiative of the Instituto de Salud Carlos III (ISCIII), Spain.

\section{Author details}

${ }^{1}$ Molecular Genetics Unit, Hospital Valdecilla, and Instituto de Formación e Investigación Marqués de Valdecilla (IFIMAV), Santander, Spain. ${ }^{2}$ HHT Spanish Unit, Hospital Sierrallana and Centro de Investigacion Biomedica en Red de Enfermedades Raras (CIBERER), Torrelavega, Santander, Spain. ${ }^{3}$ Centro de Investigaciones Biológicas, Consejo Superior de Investigaciones Científicas (CSIC), and Centro de Investigación Biomédica en Red de Enfermedades Raras (CIBERER), Madrid, Spain. ${ }^{4}$ Bioarray S.L, Crevillente, Alicante, Spain.

\section{Received: 2 April 2013 Accepted: 18 November 2013}

Published: 25 November 2013

\section{References}

1. Guttmacher AE, Marchuk DA, White RI Jr: Hereditary hemorrhagic telangiectasia. N Engl J Med 1995, 333:918-924.

2. Shovlin $\mathrm{CL}$, Guttmacher AE, Buscarini E, Faughnan ME, Hyland RH, Westermann CJ, Kjeldsen AD, Plauchu H: Diagnostic criteria for hereditary hemorrhagic telangiectasia (Rendu-Osler-Weber syndrome). Am J Med Genet 2000, 91:66-67.

3. Shovlin CL: Hereditary haemorrhagic telangiectasia: pathophysiology, diagnosis and treatment. Blood Rev 2010, 2:203-219.

4. Dakeishi M, Shioya T, Wada Y, Shindo T, Otaka K, Manabe M, Nozaki J, Inoue S, Koizumi A: Genetic epidemiology of hereditary hemorrhagic telangiectasia in a local community in the northern part of Japan. Hum Mut 2002, 19:140-148.

5. Zarrabeitia R, Albiñana V, Salcedo M, Señaris-Gonzalez B, Fernandez-Forcelledo JL, Botella LM: A review on clinical management and pharmacological therapy on hereditary haemorrhagic telangiectasia (HHT). Curr Vasc Pharmacol 2010, 8:473-481.

6. Govani FS, Shovlin CL: Hereditary haemorrhagic telangiectasia: a clinical and scientific review. Eur J Hum Genet 2009, 17:860-871.

7. McAllister KA, Grogg KM, Johnson DW, Gallione CJ, Baldwin MA, Jackson CE, Helmbold EA, Markel DS, McKinnon WC, Murrell J, et al: ENG, a TGF-beta binding protein of endothelial cells, is the gene for hereditary haemorrhagic telangiectasia type 1. Nat Genet 1994, 8:345-351.
8. Johnson DW, Berg JN, Gallione CJ, MCAllister KA, Warner JP, Helmbold EA, Markel DS, Jackson CE, Porteous ME, Marchuk DA: A second locus for hereditary hemorrhagic telangiectasia maps to chromosome 12. Genome Res 1995, 5:21-28.

9. Gallione C, Aylsworth AS, Beis J, Berk T, Bernhardt B, Clark RD, Clericuzio C, Danesino C, Drautz J, Fahl J, Fan Z, Faughnan ME, et al: Overlapping spectra of SMAD4 mutations in juvenile polyposis (JP) and JP-HHT syndrome. Am J Med Genet A 2010, 152:333-339.

10. Fernandez-L A, Sanz-Rodriguez F, Zarrabeitia R, Perez-Molino A, Morales C, Restrepo CM, Ramirez JR, Coto E, Lenato GM, Bernabeu C, Botella LM: Mutation study of Spanish patients with hereditary hemorrhagic telangiectasia and expression analysis of ENG and ALK1. Hum Mutat 2006b, 27:295.

11. Cole SG, Begbie ME, Wallace GMF, Shovlin CL: A new locus for hereditary haemorrhagic telangiactasia (HHT3) maps to chromosome 5. J Med Genet 2005, 42:577-582.

12. Bayrak-Toydemir P, McDonald J, Akarsu N, Toydemir RM, Calderon F, Tuncali T, Tang W, Miller F, Mao R: A fourth locus for hereditary hemorrhagic telangiectasia maps to chromosome 7. Am J Med Genet 2006, 140:2155-2162.

13. Santibañez JF, Quintanilla M, Bernabeu C: TGF- $\beta / T G F-\beta$ receptor system and its role in physiological and pathological conditions. Clin Sci (Lond) 2011, 121:233-251.

14. ten Dijke $\mathrm{P}$, Arthur $\mathrm{H}$ : Extracellular control of TGFbeta signalling in vascular development and disease. Nat Rev Mol Cell Biol 2007, 8:857-869.

15. Goumans MJ, Valdimarsdottir G, Itoh S, Rosendahl A, Sideras P, ten Dijke P: Balancing the activation state of the endothelium via two distinct TGF-beta type I receptors. EMBO J 2002, 21:1743-1753.

16. Lebrin F, Goumans MJ, Jonker L, Carvalho RL, Valdimarsdottir G, Thorikay M, Mummery C, Arthur HM, ten Dijke P: Endoglin promotes endothelial cell proliferation and TGF-beta/ALK1 signal transduction. EMBO J 2004, 23:4018-4028.

17. Blanco FJ, Santibanez JF, Guerrro-Esteo M, Langa C, Vary CP, Bernabeu C: Interactions and functional interplay between Endoglin and ALK-1, two components of the endothelial transforming growth factor-beta receptor complex. J Cell Physiol 2005, 204:574-584.

18. Fernández-L A, Sanz-Rodriguez F, Blanco FJ, Bernabéu C, Botella LM: Hereditary hemorrhagic telangiectasia, a vascular dysplasia affecting the TGF-beta signaling pathway. Clin Med Res 2006a, 4:66-78.

19. Pardali E, Goumans MJ, Ten Dijke P: Signaling by members of the TGF-beta family in vascular morphogenesis and disease. Trends Cell Biol 2010, 20:556-567.

20. Damjanovich K, Langa C, Blanco FJ, McDonald J, Botella LM, Bernabeu C, Wooderchak-Donahue W, Stevenson DA, Bayrak-Toydemir P: 5'UTR mutations of ENG cause hereditary hemorrhagic telangiectasia. Orphanet J Rare Dis 2011, 6:85.

21. Kim MJ, Kim ST, Lee HD, Lee KY, Seo J, Lee JB, Lee YJ, Oh SP: Clinical and genetic analyses of three Korean families with hereditary hemorrhagic telangiectasia. BMC Med Genet 2011, 12:130.

22. Fontalba A, Fernandez-L A, García-Alegria E, Albiñana V, Garrido-Martin EM Blanco FJ, Zarrabeitia R, Perez-Molino A, Bernabeu-Herrero ME, Ojeda ML, Fernandez-Luna JL, Bernabeu C, Botella LM: Mutation study of Spanish patients with hereditary hemorrhagic telangiectasia. BMC Med Genet 2008, 9:75.

23. Schouten JP, McElgunn CJ, Waaijer R, Zwijnenburg D, Diepvens F, Pals G: Relative quantification of 40 nucleic acid sequences by multiplex ligation-dependent probe amplification. Nucleic Acids Res 2002, 30:e57.

24. Ríus C, Smith JD, Almendro N, Langa C, Botella LM, Marchuk DA, Vary CP Bernabéu C: Cloning of the promoter region of human endoglin, the target gene for hereditary hemorrhagic telangiectasia type 1. Blood. 1998, 92:4677-4690

25. Richards-Yutz J, Grant K, Chao EC, Walther SE, Ganguly A: Update on molecular diagnosis of hereditary hemorrhagic telangiectasia. Hum Genet 2010, 128:61-77.

26. Prigoda NL, Savas S, Abdalla SA, Piovesan B, Rushlow D, Vandezande K Zhang E, Ozcelik H, Gallie BL, Letarte M: Hereditary haemorrhagic telangiectasia: mutation detection, test sensitivity and novel mutations. J Med Genet 2006, 43:722-728.

27. Botella LM, Sánchez-Elsner T, Rius C, Corbí A, Bernabéu C: Identification of a critical Sp1 site within the endoglin promoter and its involvement in the transforming growth factor-beta stimulation. J Biol Chem 2001, 14:34486-34494. 
28. Botella LM, Sánchez-Elsner T, Sanz-Rodriguez F, Kojima S, Shimada J, Guerrero-Esteo M, Cooreman MP, Ratziu V, Langa C, Vary CP, Ramirez JR, Friedman S, Bernabéu C: Transcriptional activation of endoglin and transforming growth factor-beta signaling components by cooperative interaction between Sp1 and KLF6: their potential role in the response to vascular injury. Blood 2002, 100:4001-4010.

29. Sánchez-Elsner T, Botella LM, Velasco B, Langa C, Bernabéu C: Endoglin expression is regulated by transcriptional cooperation between the hypoxia and transforming growth factor-beta pathways. J Biol Chem 2002, 277:43799-43808.

30. Wooderchak W, Gedge F, McDonald M, Krautscheid P, Wang X, Malkiewicz J, Bukjiok CJ, Lewis T, Bayrak-Toydemir P: Hereditary hemorrhagic telangiectasia: two distinct ENG deletions in one family. Clinical Genetics 2010, 78:484-489.

31. Deininger PL, Batzer MA: Alu repeats and human disease. Mol Genet Metab 1999, 67:183-193.

32. Sen SK, Han K, Wang J, Lee J, Wang H, Callinan PA, Dyer M, Cordaux R, Liang P, Batzer MA: Human genomic deletions mediated by recombination between Alu elements. Am J Human Genet 2006, 79:41-53.

doi:10.1186/1471-2350-14-121

Cite this article as: Fontalba et al:: Copy number variations in endoglin locus: mapping of large deletions in Spanish families with hereditary hemorrhagic telangiectasia type 1. BMC Medical Genetics 2013 14:121.

\section{Submit your next manuscript to BioMed Central and take full advantage of:}

- Convenient online submission

- Thorough peer review

- No space constraints or color figure charges

- Immediate publication on acceptance

- Inclusion in PubMed, CAS, Scopus and Google Scholar

- Research which is freely available for redistribution 